\title{
ON THE STABILITY OF ALMOST CONVEX FUNCTIONS
}

\author{
J. C. PARNAMI AND H. L. VASUDEVA
}

\begin{abstract}
Let $\mathbf{R}$ denote the set of real numbers and $I$ an open interval of R. A function $f: I \rightarrow R$ is said to be almost $\delta$-convex iff $f(t x+(1-t) y) \leq$ $t f(x)+(1-t) f(y)+\delta$ holds for all $(x, y) \in I \times I \backslash N$, where $N \subset I \times I$ is of measure zero, each $t \in[0,1]$ and some $\delta \geq 0$. It is proved that such a function is uniformly close to a convex function almost everywhere.
\end{abstract}

Let $\mathbf{R}$ be the set of all real numbers and let $I$ be an open interval (finite or infinite) in $\mathbf{R}$. A function $f: I \rightarrow \mathbf{R}$ is called convex iff

$$
f(t x+(1-t) y) \leq t f(x)+(1-t) f(y)
$$

holds for all pairs $(x, y)$ in $I \times I$ and each $t \in[0,1]$. A function $f: I \rightarrow \mathbf{R}$ is called almost convex iff (1) holds in $I \times I$ except for a set $N \subset I \times I$ of planar Lebesgue measure zero. Let $\delta$ be a nonnegative real number. A function $f: I \rightarrow \mathbf{R}$ is called $\delta$-convex iff

$$
f(t x+(1-t) y) \leq t f(x)+(1-t) f(y)+\delta
$$

for all $(x, y) \in I \times I$ and each $t \in[0,1]$. In case $\delta=0, f: I \rightarrow \mathbf{R}$ is the usual convex function. A function $f: I \rightarrow \mathbf{R}$ is called almost $\delta$-convex iff (2) holds in $I \times I \backslash N$, where $N \subset I \times I$ is of planar Lebesgue measure zero.

Is every almost convex function equal almost everywhere in $I$ (in the sense of linear Lebesgue measure) to a convex function? Is every almost $\delta$-convex function uniformly close to a convex function almost everywhere? Professor P. M. Gruber raised the former question during a conversation. In what follows we shall answer the latter question in the affirmative and obtain the answer to the former as a corollary to our theorem.

Kuczma [6] proved that an almost mid-convex function, i.e., a function $f: I \rightarrow \mathbf{R}$ satisfying

$$
f\left(\frac{x+y}{2}\right) \leq \frac{f(x)+f(y)}{2}
$$

for all pairs $(x, y)$ in $I \times I \backslash N$, where $N \subset I \times I$ is of planar Lebesgue measure zero, differs from a mid-convex function on a set of linear Lebesgue measure zero.

The question concerning the stability of convex functions has first been raised by Hyers and Ulam [4] and they proved the stability of convex functions. Cholewa [2], in a recent paper, gave a short proof of the fact that a $\delta$-convex function $f: I \rightarrow \mathbf{R}$ is uniformly close to a convex function. He also gave an example that mid-convex functions are not stable in the sense of Hyers and Ulam.

N. G. de Bruijn [1] and Jurkat [5], while answering Erdös' problem [3], proved that an almost additive function equals an additive function almost everywhere.

We state and prove a theorem on almost $\delta$-convex functions.

Received by the editors March 25, 1985.

1980 Mathematics Subject Classification. Primary 39C05.

(C) 1986 American Mathematical Society $0002-9939 / 86 \$ 1.00+\$ .25$ per page 
THEOREM. Let $f: I \rightarrow \mathbf{R}$ be a function satisfying

$$
f(t x+(1-t) y) \leq t f(x)+(1-t) f(y)+\delta
$$

for all pairs $(x, y)$ in $I \times I \backslash N$, where $N$ is a subset of $I \times I$ of planar Lebesgue measure zero, each $t \in[0,1]$ and some $\delta \geq 0$. Then there exists a convex function $h: I \rightarrow \mathbf{R}$ such that

$$
|f(x)-h(x)| \leq 3 \delta \quad \text { for all } x \in I \backslash M,
$$

where $M \subset I$ is of linear Lebesgue measure zero.

PROOF. $1^{\circ}$ For a subset $K$ of $\mathbf{R} \times \mathbf{R}$ and $x \in \mathbf{R}$, set

$$
K_{x}=\{y:(x, y) \in K\} \text { and } T(K)=\{(y, x):(x, y) \in K\} .
$$

Observe that if $K$ is of planar Lebesgue measure zero, then so is $T(K)$. Since $N \cup T(N)$ is of planar measure zero, there therefore exists a subset $M \subset I$ of linear measure zero such that $x \notin M$ implies $(N \cup T(N))_{x}$ is of measure zero. We claim that for $x, y \notin M$ and $t \in[0,1]$,

$$
f(t x+(1-t) y) \leq t f(x)+(1-t) f(y)+2 \delta
$$

holds. Let $z$ be any real number satisfying the inequality $x<z<y$. Choose $\varepsilon>0$ such that $\varepsilon<y-z$. Find $u \notin(N \cup T(N))_{y}$ and $v=v(\varepsilon) \notin(N \cup T(N))_{x}$ satisfying $x<u<z$ and $z<y-\varepsilon<v<y$, respectively. Such $u$ and $v$ exist since each of $(N \cup T(N))_{y}$ and $(N \cup T(N))_{x}$ is of measure zero. It may be noted that $u$ does not depend on $\varepsilon$ whereas $v$ does. We then have

$$
f(z) \leq \frac{v-z}{v-x} f(x)+\frac{z-x}{v-x} f(v)+\delta,
$$

since $(v, x) \notin N$ and $x<z<v$, and

$$
f(v) \leq \frac{y-v}{y-v} f(u)+\frac{v-u}{y-u} f(y)+\delta
$$

since $(u, y) \notin N$ and $u<v<y$.

Using (5) and (6), we get

$$
f(z) \leq \frac{v-z}{v-x} f(x)+\frac{z-x}{v-x}\left\{\frac{y-v}{y-u} f(u)+\frac{v-u}{y-u} f(y)+\delta\right\}+\delta .
$$

On letting $\varepsilon \rightarrow 0, v \rightarrow y$ and (7) becomes

$2^{\circ}$ Set

$$
f(z) \leq \frac{y-z}{y-x} f(x)+\frac{z-x}{y-x} f(y)+2 \delta .
$$

$$
g(x)=\inf \left(\frac{v-x}{v-u} f(u)+\frac{x-u}{v-u} f(v)\right), \quad x \in I,
$$

where inf is taken over $u, v \notin M, u \neq v$ and satisfying the inequality $u \leq x \leq v$.

It is immediate from the definition and $1^{\circ}$ that

$$
g(x) \geq f(x)-2 \delta \quad \text { for each } x \in I
$$

and

$$
g(x) \leq f(x) \quad \text { for each } x \notin M .
$$


Moreover, for $x, y \notin M, x<z<y$, on using (8), we have

$$
g(z) \leq \frac{y-z}{y-x} g(x)+\frac{z-x}{y-x} g(y)+2 \delta .
$$

We next show that $g$ is $2 \delta$-convex on $I$. Let $u<w<v$ be arbitrary in $I$ and let $\varepsilon>0$ be given. From the definition of $g$, it follows that there exist $a, b \notin M, a \neq b$, $a \leq u \leq b$ and $c, d \notin M, c \neq d, c \leq v \leq d$ such that

$$
g(u)+\varepsilon>\frac{b-u}{b-a} f(a)+\frac{u-a}{b-a} f(b)
$$

and

$$
g(v)+\varepsilon>\frac{d-v}{d-c} f(c)+\frac{v-c}{d-c} f(d) .
$$

Let $x, x^{\prime} \notin M$ satisfy $a<x<u<w<v<x^{\prime}<d$. Then

$$
g(w) \leq \frac{x^{\prime}-w}{x^{\prime}-x} g(x)+\frac{w-x}{x^{\prime}-x} g\left(x^{\prime}\right)+2 \delta,
$$

on using (10). Since $a<x<b, c<x^{\prime}<d$, on using the definition of $g$ and (13), we have

$$
\begin{aligned}
g(w) \leq & \frac{x^{\prime}-w}{x^{\prime}-x}\left\{\frac{b-x}{b-a} f(a)+\frac{x-a}{b-a} f(b)\right\} \\
& +\frac{w-x}{x^{\prime}-x}\left\{\frac{d-x^{\prime}}{d-c} f(c)+\frac{x^{\prime}-c}{d-c} f(d)\right\}+2 \delta .
\end{aligned}
$$

On letting $x \rightarrow u$ and $x^{\prime} \rightarrow v$ in (14) and using (11) and (12), we get

$$
g(w) \leq \frac{v-w}{v-u} g(u)+\frac{w-u}{v-u} g(v)+2 \delta+\varepsilon .
$$

Since $\varepsilon>0$ is arbitrary, it follows that $g: I \rightarrow \mathbf{R}$ is $2 \delta$-convex.

$3^{\circ}$ In view of a theorem of Hyers and Ulam [4, Theorem 1$]$, there exists a convex function $h: I \rightarrow \mathbf{R}$ such that

$$
|g(x)-h(x)| \leq \delta \quad \text { for each } x \in I .
$$

On using (8) and (9), we obtain

$$
|g(x)-f(x)| \leq 2 \delta \quad \text { for each } x \in I \backslash M .
$$

(15) and (16) together imply

$$
|f(x)-h(x)| \leq 3 \delta \quad \text { for each } x \in I \backslash M .
$$

COROllary. Let $f: I \rightarrow \mathbf{R}$ be a function satisfying

$$
f(t x+(1-t) f(y)) \leq t f(x)+(1-t) f(y)
$$

for all pairs $(x, y) \in I \times I \backslash N$, where $N \subset I \times I$ is of planar measure zero and each $t \in[0,1]$. Then there exists a unique convex function $h: I \rightarrow \mathbf{R}$ such that $f(x)=h(x)$ for each $x \in I \backslash M$, where $M \subset I$ is of linear measure zero.

PROOF. The existence of $h$ follows on taking $\delta=0$ in the Theorem above. The facts that a convex function defined on an open interval is continuous and a continuous function which equals zero almost everywhere on an interval is identically zero imply the uniqueness. 


\section{REFERENCES}

1. N. G. de Bruijn, On almost additive functions, Colloq. Math. 15 (1966), 59-63.

2. P. W. Cholewa, Remarks on the stability of functional equations, Aequationes Math. 27 (1984), 76-86.

3. P. Erdös, Colloq. Math. 7 (1960), 311.

4. D. H. Hyers and S. M. Ulam, Approximately convex functions, Proc. Amer. Math. Soc. 3 (1952), 821-828.

5. W. B. Jurkat, On Cauchy's functional equation, Proc. Amer. Math. Soc. 16 (1965), 683686.

6. M. Kuczma, Almost convex functions, Colloq. Math. 21 (1970), 279-284.

Department of Mathematics, PANJab University, Chandigarh-160014, INDia 